\title{
Thermal diffusion of oligosaccharide solutions: the role of chain length and structure
}

\author{
Pablo Blanco, ${ }^{*}$ Hartmut Kriegs, Bastian Arlt, and Simone Wiegand* \\ Forschungszentrum Jülich GmbH, IFF - Soft Matter, D-52428 Jülich, Germany \\ E-mail: p.blanco@fz-juelich.de; s.wiegand@fz-juelich.de
}

*To whom correspondence should be addressed 


\begin{abstract}
We investigated the chain length dependence of the thermodiffusion behavior of oligosaccharides by the infrared thermal diffusion forced Rayleigh scattering (IR-TDFRS) technique. Three disaccharides: sucrose, cellobiose and maltose, two trisaccharides: melezitose and raffinose, and a tetrasaccharide: stachyose have been studied. We determined the thermal diffusion $\left(D_{\mathrm{T}}\right)$, mass diffusion $(D)$ and Soret $\left(S_{\mathrm{T}}\right)$ coefficient as function of temperature and concentration. While monosaccharides in water accumulate at the cold side in the investigated temperature $\left(20^{\circ} \mathrm{C}\right.$ to $\left.50{ }^{\circ} \mathrm{C}\right)$ and concentration $(0.2 \mathrm{wt}$ to $0.6667 \mathrm{wt})$ range, oligosaccharides enrich on the warm side with decreasing temperature or increasing sugar concentration. Additionally, we determined the kinematic viscosity $(v)$, the density $(\rho)$ and the thermal expansion coefficient $(\alpha)$ of the mixtures in order to check the linear correlation between $D_{\Gamma}$ and the ratio $\alpha / \nu$, which has been recently found for aqueous solutions of monosaccharides and for alkane mixtures. Finally, we found that $D_{\mathrm{T}}$ and $D$ decay with increasing chain length of the oligosaccharides in the whole studied range of temperatures, in contradiction with experimental results for non-polar mixtures and theoretical predictions.
\end{abstract}

July 14,2010

\title{
Introduction
}

The consequence of applying a temperature gradient to an initially homogeneous mixture is a mass separation of its constituents. This mass separation depends on the thermal diffusion properties: (a) the thermal diffusion coefficient $\left(D_{\mathrm{T}}\right)$ which attempts to separate its components, and (b) the mass diffusion coefficient $(D)$ which aims to homogenize the concentration of the mixture. In equilibrium, both contributions compensate each other, resulting in a zero net flux. In binary mixtures, the Soret coefficient $\left(S_{\mathrm{T}}=D_{\mathrm{T}} / D\right)$ represents the mass separation under the applied temperature gradient. The measure of the mass separation (Soret effect) will depend on the tendency of a component to either go to cold or to warm regions (thermal diffusion) and the velocity of the components to balance their concentration (mass diffusion). We use the convention that in a binary mixture of $A$ 
and $B$, the value of $S_{\mathrm{T}}$ for $A$, the first named component, is positive if $A$ moves to the cold side. This implies that the $S_{\mathrm{T}}$ of $B$ is negative, because $B$ migrates to the warm side. The same holds for $D_{\mathrm{T}}{ }^{1}$.

A physical interpretation of the molecular transport phenomenon of thermal diffusion or thermodiffusion cannot yet be provided. Although it was first described more than 150 years ago, it is still one of the unresolved challenges in the field of physical chemistry.

In a recent study ${ }^{2}$ the thermal diffusion phenomena in aqueous solutions of monosaccharides have been investigated. As in the case of branched alkanes, ${ }^{3}$ these systems allow a systematic variation of the molecule shape, while the molecular weight is identical. It turned out that for a given temperature and concentration, the mass diffusion coefficient was the same for all studied monosaccharides. The thermal diffusion and Soret coefficients were positive in the investigated temperature and concentration range, which means that the sugar molecules move to cold region, while water molecules enrich on the warm side. At low sugar concentration the thermal diffusion coefficient depends linearly on the ratio between the thermal expansion and kinematic viscosity of the mixtures. The same correlation had been found earlier by Blanco et al. ${ }^{4}$ for equimolar mixtures of normal alkanes. In this work we extend our previous study ${ }^{2}$ to oligosaccharides in water in order to investigate the influence of the molecular length on the thermal diffusion properties, as has been done in alkane binary mixtures ${ }^{4-8}$ and polymer solutions. ${ }^{9-12}$

Oligosaccharides are biomolecules of crucial interest in biology, as they are present in many fundamental processes including immune defence, fertilisation, viral replication, parasite infection, cell growth, cell-cell adhesion, degradation of blood clots, and inflammation. ${ }^{13}$ Oligosaccharides consist of a small number of sugar monomers (monosaccharides). Due to the many different monosaccharide combinations, their number of units and linkage between them, there is a large variety of oligosaccharides. As a consequence of the huge diversity of oligosaccharides, they are considered to be well-suited for storage of information. For example, they fulfil the task of cell recognition. In nature, they are found in the external cover of the cell membrane, linked to protein and lipid molecules, forming glycoproteins and glycolipids. Their function is to provide the cell 
with an identity signal, so the different cell types can recognize each other by the oligosaccharides present on the outside of their membranes.

The infrared thermal diffusion forced Rayleigh scattering (IR-TDFRS) technique ${ }^{14}$ has been used to determine the mass diffusion, thermal diffusion and Soret coefficients of six oligosaccharides in aqueous solutions. Specifically, three disaccharides: sucrose, maltose and cellobiose; two trisaccharides: melezitose and raffinose; and a tetrasaccharide: stachyose. The main objective od this work is to study the effect of the sugar molecule's length in the thermal diffusion properties. Additionally, the influence of the mass concentration of the sugar and temperature in the thermal diffusion, mass diffusion and Soret coefficients is shown. As in the previous study, ${ }^{2}$ we discuss the physical principles which correlate the thermal diffusion behavior with other thermophysical properties such as viscosity and thermal expansion.

\section{Experimental section}

\section{Infrared Thermal Diffusion Forced Rayleigh Scattering}

A detailed description of the thermal diffusion forced Rayleigh scattering technique can be found

in the literature. ${ }^{1,15-19}$ We have used the infrared thermal diffusion forced Rayleigh scattering (IR-TDFRS) setup, ${ }^{14}$ which slightly differs from the classical setup. The main difference is that no dye is needed for aqueous mixtures to convert the light energy into heat energy, due to the absorption of water at the wavelength of the infrared laser beam $\left(\lambda_{w}=980 \mathrm{~nm}\right)$. Summarizing what is explained in more detail elsewhere, the infrared laser beam is split into two beams of equal intensity. Thes beams interfere in the sample cell, creating an intensity grating which is transformed into a temperature grating and then into a concentration grating due to the thermal diffusion. Both temperature and concentration gratings lead to a combined refractive index grating which is read out under the Bragg angle by a He-Ne laser $\left(\lambda_{w}=633 \mathrm{~nm}\right)$.

The total intensity $\zeta_{\text {het }}(t)$ normalized to the thermal signal is given by 


$$
\zeta_{\text {het }}(t)=1+\frac{(\partial n / \partial c)_{p, T}}{(\partial n / \partial T)_{p, c}} S_{\mathrm{T}} c(1-c)\left(1-e^{-q^{2} D t}\right)
$$

where $c$ is the mass concentration, $(\partial n / \partial c)_{p, T}$ and $(\partial n / \partial T)_{p, c}$ are, respectively, the contrast factors of the refractive index increment as a function of the mass concentration at constant pressure and temperature, and the refractive index increment as a function of the temperature at constant pressure and mass concentration. The magnitude of the grating vector, $q$, is given by

$$
q=\frac{4 \pi}{\lambda_{w}} \sin \frac{\theta}{2}
$$

where $\theta$ is the angle between the two writing beams at the wavelength $\lambda_{w}$. The transport coefficients are determined by fitting Eq. (1) to the measured heterodyne signal and deconvoluting the excitation function. ${ }^{20}$

\section{Materials and Equipment}

Sucrose $(\geq 99.5 \%)$, D-(+)-cellobiose ( $\geq 99 \%)$, D-(+)-maltose monohydrate $(\geq 99 \%)$, D-(+)-melezitose monohydrate $(\geq 99 \%)$, D-(+)-raffinose pentahydrate $(\geq 99 \%)$ and stachyose hydrate $(\geq 98 \%)$ were purchased from Sigma-Aldrich. In Figure 1 the structure of the different oligosaccharides is shown. Deionized water from a Millipore filter unit $(0.22 \mu \mathrm{m})$ was used.

A Mettler Toledo XP504 digital scale with an accuracy of $0.0001 \mathrm{~g}$ was used to prepare by weighing the mass concentration of the samples. The samples were filtered through a $0.45 \mu \mathrm{m}$ filter (Spartam 13/0.45 RC) before filling an optical quartz cell (Hellma) which has an optical path length of $0.2 \mathrm{~mm}$. The experiments were repeated, at least three times for each temperature and concentration of the studied mixtures.

The dynamic viscosity of the mixtures, $\eta$, was measured by means of an Anton Paar AMVn microviscometer with a reproducibility $<0.1 \%$. The temperature resolution is $\Delta T=0.01 \mathrm{~K}$ and the time resolution is of the order of $0.001 \mathrm{~s}$. Two different capillaries were used to measure the viscosities of the mixtures with sugar mass fractions of $c=0.1-0.4$ and of $c=0.6667$. The 
first capillary was calibrated with water from the Millipore filter unit. The second capillary was calibrated with a viscosity standard (20 BW) purchased from ZMK-ANALYTIK-GmbH. Each viscosity measurement was repeated eight times at seven different angles (from $20^{\circ}$ to $80^{\circ}$ ).

The density, $\rho$, as well as the thermal expansion coefficient, $\alpha$, of the mixtures were determined by means of an Anton Paar DMA 4500 densimeter, which has an accuracy of $\Delta \rho= \pm 0.00001$ $\mathrm{gcm}^{-3}$ and a temperature control $\Delta T= \pm 0.01 \mathrm{~K}$.

The contrast factors $(\partial n / \partial c)_{p, T}$ of the mixtures were determined with an Anton Paar RXA 156 refractometer around the desired concentration $(\bar{c} \pm 0.02)$. The temperature control is $\Delta T= \pm 0.01$ $\mathrm{K}$ and the accuracy is $\Delta n=0.00003$. A Michelson interferometer was used to determine the refractive index increments with temperature $(\partial n / \partial T)_{p, c}$ around the desired temperature $( \pm 2 \mathrm{~K})$. A full description of this setup is described elsewhere. ${ }^{21}$ In all cases, a linear dependence of the refractive index with mass concentration or temperature was found.

The studied mixtures are listed in Table 1 and Table 2, together with $\alpha, \rho, \eta$ and the contrast factors $(\partial n / \partial c)_{p, T}$ and $(\partial n / \partial T)_{p, c}$.

\section{Results and Discussion}

\section{Thermal diffusion properties of different oligosaccharide-water solutions: in-}

\section{fluence of the molecule length}

Six different oligosaccharide aqueous solutions with a sugar weight fraction of $c=0.1$ were studied at five different temperatures, from $15^{\circ} \mathrm{C}$ to $50{ }^{\circ} \mathrm{C}$. Together with the results of the monosaccharide aqueous solution of glucose ${ }^{2}$ we studied the influence of the molecule length in the thermal diffusion properties. The values of thermal diffusion, mass diffusion and Soret coefficients are listed in Table 3 and Table 4. In general the deviation of the results for the thermal diffusion properties is within a few percent. Only a few mixtures present high deviations in the diffusion coefficient at a temperature near the Soret sign change, for instance, maltose or sucrose at $20{ }^{\circ} \mathrm{C}$. This can be explained by the small amplitude of the concentration plateau in the obtained heterodyne signal. 
Table 1: Thermophysical properties of sucrose/water mixtures at different concentrations and temperatures

\begin{tabular}{|c|c|c|c|c|c|c|c|}
\hline sugar & $\begin{array}{c}\text { sugar } \\
\text { mass } \\
\text { concentration } c\end{array}$ & $\begin{array}{c}\text { temperature } \\
T /{ }^{\circ} \mathrm{C}\end{array}$ & $\partial n / \partial c$ & $\begin{array}{c}\partial n / \partial T \\
/ 10^{-4} \mathrm{~K}^{-1}\end{array}$ & $\begin{array}{c}\text { thermal } \\
\text { expansion } \\
\alpha / 10^{-4} \mathrm{~K}^{-1}\end{array}$ & $\begin{array}{c}\text { mixture } \\
\text { density } \\
\rho / \mathrm{gcm}^{-3}\end{array}$ & $\begin{array}{r}\text { dynamic } \\
\text { viscosity } \\
\eta / \mathrm{mPas}\end{array}$ \\
\hline \multirow{4}{*}{ sucrose } & \multirow{4}{*}{0.6667} & 60 & 0.240 & -2.203 & 4.65 & 1.30456 & 21.49 \\
\hline & & 50 & 0.239 & -2.122 & 4.43 & 1.31056 & 31.58 \\
\hline & & 40 & 0.242 & -2.037 & 4.22 & 1.31626 & 56.05 \\
\hline & & 30 & 0.240 & -1.947 & 3.13 & 1.32166 & 103.8 \\
\hline \multirow{5}{*}{ sucrose } & \multirow{5}{*}{0.4} & 50 & 0.189 & -1.977 & 4.60 & 1.16243 & 2.450 \\
\hline & & 40 & 0.190 & -1.828 & 4.27 & 1.16764 & 3.204 \\
\hline & & 30 & 0.191 & -1.666 & 3.84 & 1.17240 & 4.348 \\
\hline & & 20 & 0.192 & -1.491 & 3.39 & 1.17665 & 6.143 \\
\hline & & 15 & 0.194 & -1.406 & 3.14 & 1.17857 & 7.434 \\
\hline \multirow{5}{*}{ sucrose } & \multirow{5}{*}{0.2} & 50 & 0.164 & -1.831 & 4.63 & 1.06878 & 0.956 \\
\hline & & 40 & 0.165 & -1.644 & 4.08 & 1.07373 & 1.179 \\
\hline & & 30 & 0.165 & -1.425 & 3.50 & 1.07781 & 1.491 \\
\hline & & 20 & 0.167 & -1.167 & 2.77 & 1.08120 & 1.943 \\
\hline & & 15 & 0.168 & -1.027 & 2.40 & 1.08261 & 2.248 \\
\hline \multirow{5}{*}{ sucrose } & \multirow{5}{*}{0.1} & 50 & 0.151 & -1.766 & 4.62 & 1.02703 & 0.699 \\
\hline & & 40 & 0.151 & -1.555 & 3.98 & 1.03144 & 0.848 \\
\hline & & 30 & 0.153 & -1.318 & 3.26 & 1.03519 & 1.051 \\
\hline & & 20 & 0.154 & -1.034 & 2.42 & 1.03814 & 1.342 \\
\hline & & 15 & 0.155 & -0.868 & 1.95 & 1.03929 & 1.535 \\
\hline
\end{tabular}


Table 2: Thermophysical properties of different polysugar/water mixtures at a sugar mass concentration of $c=0.1$ and at different temperatures

\begin{tabular}{c|c|c|c|c|c|c}
\hline \hline \multirow{3}{*}{ sugar } & $\begin{array}{c}\text { temperature } \\
T /{ }^{\circ} \mathrm{C}\end{array}$ & $\partial n / \partial c$ & $\begin{array}{c}\partial n / \partial T \\
/ 10^{-4} \mathrm{~K}^{-1}\end{array}$ & $\begin{array}{c}\text { thermal } \\
\text { expansion } \\
\alpha / 10^{-4} \mathrm{~K}^{-1}\end{array}$ & $\begin{array}{c}\text { mixture } \\
\text { density } \\
\rho / \mathrm{gcm}^{-3}\end{array}$ & $\begin{array}{c}\text { dynamic } \\
\text { viscosity } \\
\eta / \mathrm{mPas}\end{array}$ \\
\hline \multirow{5}{*}{ cellobiose } & 50 & 0.153 & -1.777 & 4.69 & 1.02668 & 0.726 \\
& 40 & 0.155 & -1.564 & 4.02 & 1.03110 & 0.883 \\
& 30 & 0.156 & -1.338 & 3.29 & 1.03485 & 1.097 \\
& 20 & 0.157 & -1.061 & 2.46 & 1.03784 & 1.399 \\
& 15 & 0.158 & -0.893 & 2.00 & 1.03900 & 1.595 \\
\hline \multirow{5}{*}{ maltose } & 50 & 0.154 & -1.762 & 4.51 & 1.02738 & 0.710 \\
& 40 & 0.155 & -1.558 & 3.99 & 1.03182 & 0.860 \\
& 30 & 0.156 & -1.330 & 3.31 & 1.03561 & 1.067 \\
& 20 & 0.158 & -1.052 & 2.47 & 1.03862 & 1.360 \\
& 15 & 0.159 & -0.913 & 2.04 & 1.03980 & 1.557 \\
\hline \multirow{5}{*}{ melezitose } & 50 & 0.152 & -1.759 & 4.47 & 1.02725 & 0.707 \\
& 40 & 0.153 & -1.543 & 3.87 & 1.03158 & 0.857 \\
& 30 & 0.154 & -1.301 & 3.18 & 1.03529 & 1.063 \\
& 20 & 0.155 & -1.021 & 2.37 & 1.03820 & 1.357 \\
& 15 & 0.156 & -0.865 & 1.92 & 1.03931 & 1.553 \\
\hline \multirow{5}{*}{ raffinose } & 50 & 0.155 & -1.766 & 4.57 & 1.02798 & 0.721 \\
& 40 & 0.156 & -1.557 & 3.97 & 1.03249 & 0.875 \\
& 30 & 0.157 & -1.320 & 3.27 & 1.03625 & 1.083 \\
& 20 & 0.159 & -1.043 & 2.42 & 1.03921 & 1.383 \\
& 15 & 0.160 & -0.878 & 2.01 & 1.04035 & 1.585 \\
\hline & 50 & 0.159 & -1.764 & 4.62 & 1.02893 & 0.745 \\
& 40 & 0.160 & -1.555 & 3.99 & 1.02337 & 0.903 \\
& 30 & 0.161 & -1.319 & 3.26 & 1.03713 & 1.119 \\
& 20 & 0.163 & -1.043 & 2.43 & 1.04011 & 1.430 \\
& 15 & 0.164 & -0.879 & 1.89 & 1.04126 & 1.638 \\
\hline \multirow{5}{*}{} & 50 & & & & &
\end{tabular}


Table 3: Thermal diffusion, mass diffusion and Soret coefficients of sucrose/water mixtures at different concentrations and temperatures

\begin{tabular}{c|c|c|c|c|c}
\hline \hline \multirow{2}{*}{ sugar } & $\begin{array}{c}\text { sugar mass } \\
\text { concentration }\end{array}$ & $\begin{array}{c}\text { temperature } \\
/{ }^{\circ} \mathrm{C}\end{array}$ & $\begin{array}{c}S_{\mathrm{T}} \\
/ 10^{-3} \mathrm{~K}^{-1}\end{array}$ & $\begin{array}{c}D \\
/ 10^{-10} \mathrm{~m}^{2} \mathrm{~s}^{-1}\end{array}$ & $\begin{array}{c}D_{T} \\
/ 10^{-12} \mathrm{~m}^{2} \mathrm{~s}^{-1} \mathrm{~K}^{-1}\end{array}$ \\
\hline \multirow{3}{*}{ sucrose } & \multirow{3}{*}{0.6667} & 60 & $0.67 \pm 0.01$ & $2.67 \pm 0.20$ & $0.18 \pm 0.01$ \\
& & 50 & $-0.03 \pm 0.00$ & $2.20 \pm 0.61$ & $-0.01 \pm 0.00$ \\
& & 40 & $-0.90 \pm 0.02$ & $1.42 \pm 0.03$ & $-0.13 \pm 0.00$ \\
& & 50 & $-2.05 \pm 0.01$ & $0.94 \pm 0.01$ & $-0.19 \pm 0.00$ \\
\hline \multirow{5}{*}{ sucrose } & \multirow{3}{*}{0.4} & 40 & $2.59 \pm 0.02$ & $4.77 \pm 0.06$ & $1.24 \pm 0.02$ \\
& & 30 & $0.64 \pm 0.01$ & $2.76 \pm 0.10$ & $0.18 \pm 0.01$ \\
& & 20 & $-0.87 \pm 0.01$ & $2.03 \pm 0.06$ & $-0.18 \pm 0.00$ \\
& & 15 & $-1.82 \pm 0.02$ & $1.70 \pm 0.02$ & $-0.31 \pm 0.00$ \\
\hline \multirow{5}{*}{ sucrose } & \multirow{3}{*}{0.2} & 40 & $3.14 \pm 0.03$ & $7.14 \pm 0.36$ & $2.24 \pm 0.10$ \\
& & 30 & $2.35 \pm 0.03$ & $5.82 \pm 0.41$ & $1.36 \pm 0.08$ \\
& & 20 & $-0.17 \pm 0.01$ & $2.44 \pm 0.90$ & $-0.04 \pm 0.01$ \\
& & 15 & $-1.06 \pm 0.01$ & $2.68 \pm 0.09$ & $-0.28 \pm 0.01$ \\
\hline \multirow{3}{*}{ sucrose } & \multirow{3}{*}{0.1} & 50 & $3.15 \pm 0.01$ & $8.97 \pm 0.81$ & $2.83 \pm 0.25$ \\
& & 40 & $2.37 \pm 0.05$ & $7.09 \pm 0.48$ & $1.68 \pm 0.12$ \\
& & 30 & $1.31 \pm 0.03$ & $5.62 \pm 0.91$ & $0.73 \pm 0.12$ \\
& & 20 & $-0.15 \pm 0.03$ & $2.69 \pm 2.25$ & $-0.40 \pm 0.37$ \\
& & 15 & $-1.06 \pm 0.01$ & $3.34 \pm 0.60$ & $-0.35 \pm 0.06$ \\
\hline \hline
\end{tabular}


In Figure 2 the thermal diffusion properties at $50{ }^{\circ} \mathrm{C}$ as functions of the number of monomers are shown. The values of glucose have been extrapolated from the mass concentration dependence at $50{ }^{\circ} \mathrm{C}$ (cf. figure 5 of $\operatorname{ref}^{2}$ ).

The thermal diffusion coefficient of the sugars decreases with increasing size of the sugar molecule (molar mass) for all investigated temperatures. A similar trend has been found for DNA where $D_{\mathrm{T}}$ decreases with the number of base pairs of DNA. ${ }^{22}$ Contrary experimental results have been found for non polar polymer solutions, where $D_{\mathrm{T}}$ increases with the molar mass of polystyrene. ${ }^{12}$ Additionally, the theoretical approach by Würger predicts the opposite chain length dependence. ${ }^{10}$ In this model it is assumed that all "atoms" interact through van der Waals forces, and specific interactions present in polar mixtures are not considered.

If we consider the magnitude of $D_{\mathrm{T}}$, the behavior of 1-methylnaphthalene-alkane mixtures ${ }^{7}$ and oligosaccharides-water solutions at higher temperatures look similar, because in both cases the magnitude of $D_{\mathrm{T}}$ decreases with increasing molar mass (c.f. Figure 2). On the other hand, in alkane-alkane mixtures ${ }^{5-8,23}$ the magnitude of $D_{\mathrm{T}}$ becomes smaller with decreasing mass difference, which is the same trend as for the oligosaccharides in water at lower temperatures $\left(15^{\circ} \mathrm{C}\right.$, cf Table 4). This is not the case for alkanes in 1-methylnaphthalene because the magnitude of $D_{\mathrm{T}}$ decreases monotonically and does not show a minimum for decane, which has almost the same mass as 1-methylnaphthalene. In the case of the oligosaccharides at higher temperatures, a larger mass difference between the oligosaccharide and water leads to a smaller magnitude of $D_{\mathrm{T}}$, which is opposite to the trend of the alkane/alkane mixtures.

At high temperatures the oligosaccharide-water systems behave "normally" in the sense that the heavier component enriches in the cold side, but with an increase of the molar mass or decrease of the temperature the thermophility of the sugars becomes more pronounced. Previously, Sugaya et al. ${ }^{24}$ observed that the polysaccharide dextran accumulates at the warm side for temperatures below $45^{\circ} \mathrm{C}$. This shows that in the case of aqueous solutions the contribution due to specific interactions can be more important than the mass difference, especially at low temperatures, where the hydrogen bonds play an important role. 
At a defined temperature the thermal diffusion coefficient of the three disaccharides agree within a maximum deviation of $\pm 0.110^{-12} \mathrm{~m}^{2} \mathrm{~s}^{-1} \mathrm{~K}^{-1}$, which is in the order of the uncertainty of a measurement. The same observation has been made for the two trisaccharides. Here the maximum deviation of the mean value is $\pm 0.210^{-12} \mathrm{~m}^{2} \mathrm{~s}^{-1} \mathrm{~K}^{-1}$.

The mass diffusion coefficient, $D$, decreases linearly as function of the number of monomers for sugar mixtures at $50{ }^{\circ} \mathrm{C}$ (cf. Figure 2). At lower temperatures $D$ also decreases with the number of monomers (cf. Table 3 and Table 4). A similar tendency has been found in hydrocarbon binary mixtures. ${ }^{8}$ This observation fits with the general physical picture that small molecules diffuse faster than big ones. Sugars with the same number of monomers have roughly the same mass diffusion coefficient as observed for aqueous solutions of monosaccharides. ${ }^{2}$

The Soret coefficient at $50{ }^{\circ} \mathrm{C}$ is almost independent of the number of monomers of the sugar molecule, as can be seen in Figure 2. The average value is $3.35 \times 10^{-3} \mathrm{~K}^{-1}$ with a standard deviation of $\pm 0.23 \times 10^{-3} \mathrm{~K}^{-1}$, which corresponds to $7 \%$ and is within the uncertainty of the measurement. At lower temperatures, the Soret coefficient becomes more negative with increasing number of monomers (cf. Table 3 and Table 4). As an example, in Figure 3 we have plotted the Soret coefficient as function of the number of monomers at $15^{\circ} \mathrm{C}$. In a recent study of polystyrene particle suspensions in a TRIS-HCl buffer, a similar trend has been found, where the slope of $S_{\mathrm{T}}$ versus the particle radius changes as function of the temperature. ${ }^{25}$

In aqueous solutions of monosaccharides we found, for low concentrated sugar solutions, a linear dependence of the thermal diffusion coefficient as a function of the ratio between the thermal expansion coefficient and the kinematic viscosity $\alpha / v .^{2}$ In order to check the validity for aqueous oligosaccharide solutions, the viscosity and thermal expansion of all studied mixtures have been determined. A similar trend to the case of monosaccharides ${ }^{2}$ and equimolar alkane mixtures ${ }^{4}$ can be observed in Figure 4. At lower temperatures, $D_{\mathrm{T}}$ of cellobiose deviates from the linear behavior. To our knowledge, there are no systematic studies on physical properties of all investigated sugars, so we can only speculate about the reasons for this behavior. Compared with the other sugars the rings of cellobiose are almost coplanar. ${ }^{26}$ This structural difference and the internal hy- 
Table 4: Thermal diffusion, mass diffusion and Soret coefficients of different polysugar/water mixtures at a sugar mass concentration of $c=0.1$ and at different temperatures

\begin{tabular}{|c|c|c|c|c|}
\hline sugar & $\begin{array}{c}\text { temperature } \\
{ }^{\circ} \mathrm{C}\end{array}$ & $\begin{array}{c}S_{\mathrm{T}} \\
/ 10^{-3} \mathrm{~K}^{-1}\end{array}$ & $\begin{array}{c}D \\
/ 10^{-10} \mathrm{~m}^{2} \mathrm{~s}^{-1}\end{array}$ & $\begin{array}{c}D_{T} \\
/ 10^{-12} \mathrm{~m}^{2} \mathrm{~s}^{-1} \mathrm{~K}^{-1}\end{array}$ \\
\hline \multirow{5}{*}{ cellobiose } & 50 & $3.21 \pm 0.03$ & $8.62 \pm 0.43$ & $2.76 \pm 0.12$ \\
\hline & 40 & $2.36 \pm 0.01$ & $7.18 \pm 0.29$ & $1.70 \pm 0.07$ \\
\hline & 30 & $1.28 \pm 0.01$ & $5.97 \pm 0.16$ & $0.77 \pm 0.02$ \\
\hline & 20 & $-0.23 \pm 0.02$ & $1.70 \pm 0.37$ & $-0.04 \pm 0.01$ \\
\hline & 15 & $-1.19 \pm 0.01$ & $2.84 \pm 0.15$ & $-0.34 \pm 0.02$ \\
\hline \multirow{5}{*}{ maltose } & 50 & $3.23 \pm 0.04$ & $8.21 \pm 0.11$ & $2.65 \pm 0.04$ \\
\hline & 40 & $2.51 \pm 0.02$ & $6.52 \pm 0.21$ & $1.64 \pm 0.06$ \\
\hline & 30 & $1.49 \pm 0.01$ & $5.24 \pm 0.20$ & $0.78 \pm 0.03$ \\
\hline & 20 & $0.03 \pm 0.05$ & $2.38 \pm 1.98$ & $0.01 \pm 0.01$ \\
\hline & 15 & $-0.90 \pm 0.02$ & $3.24 \pm 0.13$ & $-0.29 \pm 0.01$ \\
\hline \multirow{5}{*}{ melezitose } & 50 & $3.03 \pm 0.25$ & $7.51 \pm 0.37$ & $2.28 \pm 0.30$ \\
\hline & 40 & $2.29 \pm 0.08$ & $6.02 \pm 0.37$ & $1.38 \pm 0.11$ \\
\hline & 30 & $1.01 \pm 0.04$ & $4.79 \pm 0.47$ & $0.49 \pm 0.06$ \\
\hline & 20 & $-0.79 \pm 0.08$ & $3.04 \pm 0.38$ & $-0.24 \pm 0.03$ \\
\hline & 15 & $-1.94 \pm 0.09$ & $2.78 \pm 0.13$ & $-0.54 \pm 0.02$ \\
\hline \multirow{5}{*}{ raffinose } & 50 & $3.59 \pm 0.01$ & $7.12 \pm 0.17$ & $2.55 \pm 0.06$ \\
\hline & 40 & $2.58 \pm 0.03$ & $5.77 \pm 0.28$ & $1.49 \pm 0.08$ \\
\hline & 30 & $1.18 \pm 0.02$ & $4.92 \pm 0.23$ & $0.58 \pm 0.02$ \\
\hline & 20 & $-0.69 \pm 0.02$ & $2.67 \pm 0.16$ & $-0.18 \pm 0.01$ \\
\hline & 15 & $-1.89 \pm 0.06$ & $2.66 \pm 0.07$ & $-0.50 \pm 0.02$ \\
\hline \multirow{5}{*}{ stachyose } & 50 & $3.80 \pm 0.21$ & $5.96 \pm 0.40$ & $2.26 \pm 0.10$ \\
\hline & 40 & $2.65 \pm 0.12$ & $4.70 \pm 0.28$ & $1.24 \pm 0.04$ \\
\hline & 30 & $1.05 \pm 0.04$ & $3.83 \pm 0.51$ & $0.40 \pm 0.05$ \\
\hline & 20 & $-1.16 \pm 0.09$ & $2.69 \pm 0.09$ & $-0.31 \pm 0.30$ \\
\hline & 15 & $-2.53 \pm 0.15$ & $2.47 \pm 0.08$ & $-0.62 \pm 0.04$ \\
\hline
\end{tabular}



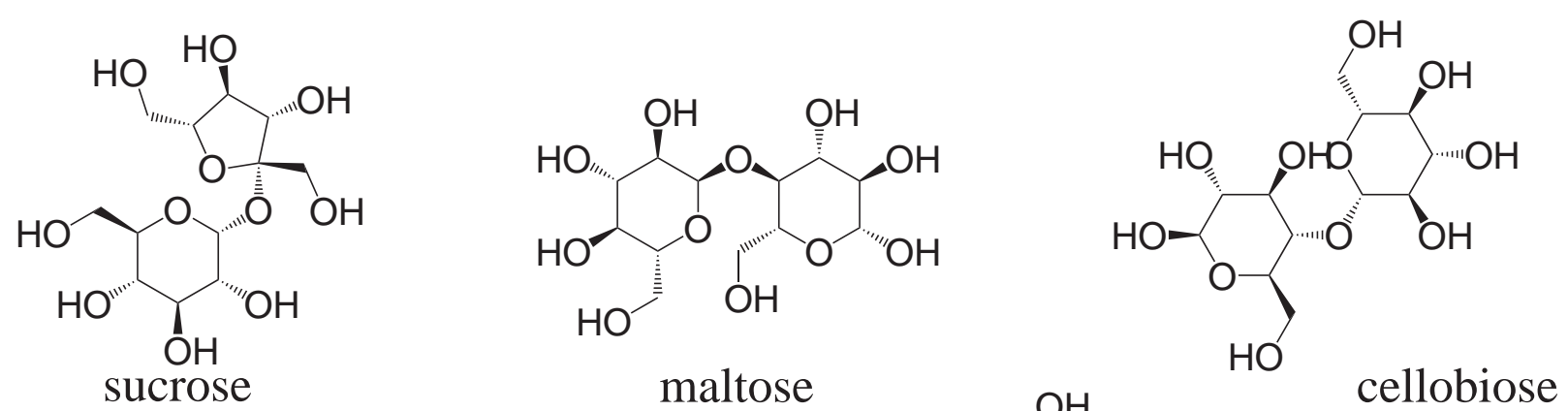

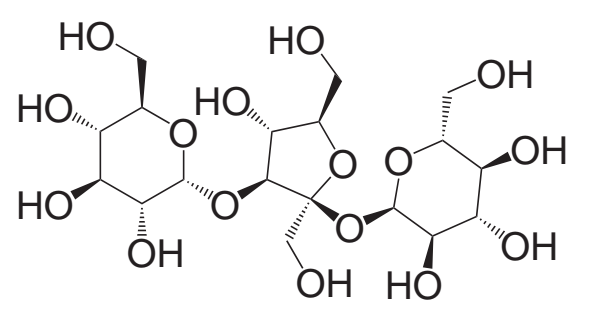

melezitose<smiles></smiles>

raffinose

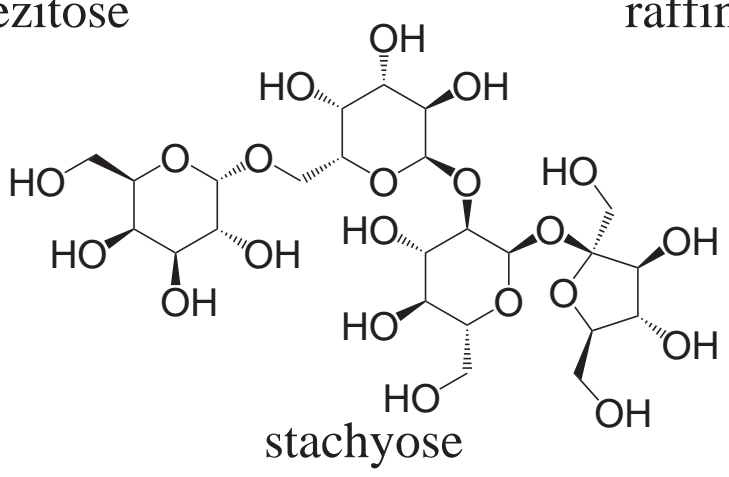

Figure 1: Natta projection of the chemical structure of all investigated oligosaccharides. 


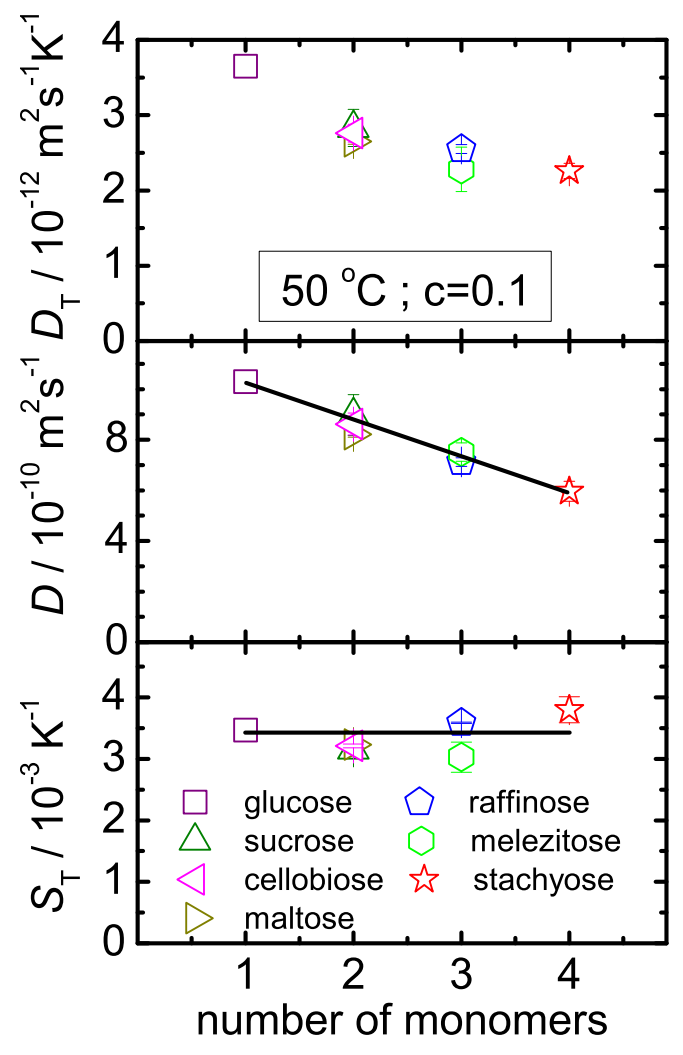

Figure 2: (color online) Thermal diffusion, mass diffusion and Soret coefficients as function of the number of monomers for different aqueous sugar solutions at $50{ }^{\circ} \mathrm{C}$ and $c=0.1$. The results of glucose have been extrapolated from Ref. ${ }^{2}$

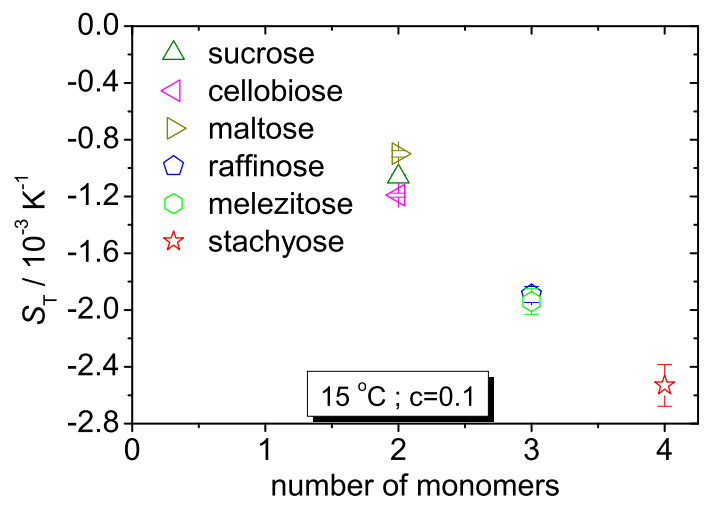

Figure 3: (color online) Thermal diffusion, mass diffusion and Soret coefficients as function of the number of monomers for different aqueous sugar solutions at $15{ }^{\circ} \mathrm{C}$ and $c=0.1$. 
drogen bonds are probably the reason for its low solubility in water and might also be responsible for the observed deviations at low temperatures. At $50{ }^{\circ} \mathrm{C}$ melezitose slightly deviates from the linear trend. If we consider the sugar series sucrose, raffinose, melezitose and stachyose, raffinose and stachyose can be regarded as chemical extensions of sucrose, while the fructofuranosyl ring of melezitose is substituted by two monosaccharide rests. Therefore, sterical hindrances can be expected, which might be responsible for the strong crystallization tendency of melezitose observed in honeydews. Finally, we conclude that, except for the aforementioned deviations, the linear dependence of $D_{\mathrm{T}}$ with the ratio $\alpha / v$ is confirmed for the oligosacharides. Different theoretical models can be found in the literature to describe the thermophoretic mobility or thermal diffusion coefficient where the thermal expansion and viscosity is directly linked to $D_{\mathrm{T}}$. For instance, in the model developed by Semenov and Schimpf for dilute solutions of dissolved or suspended components, the thermophoretic mobility $\left(D_{\mathrm{T}}\right)$ is proportional to the ratio between the thermal expansion and the dynamic viscosity. ${ }^{27}$ Likewise, Brenner's ${ }^{28}$ kinematic model of thermal diffusion for liquid and gases establishes that $D_{\mathrm{T}}$ is proportional to the thermal expansion coefficient.

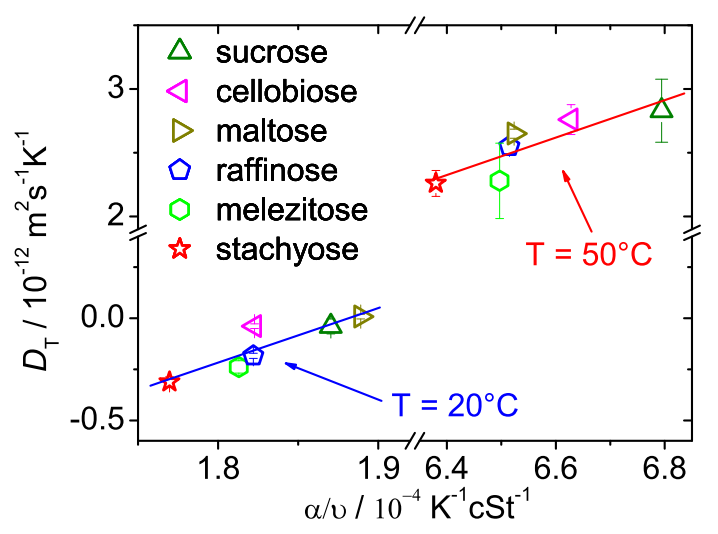

Figure 4: (color online) Thermal diffusion coefficient of oligosaccharide aqueous solutions with a sugar weight fraction $c=0.1$ at $20{ }^{\circ} \mathrm{C}$ and $50{ }^{\circ} \mathrm{C}$ as a function of the ratio of the thermal expansion coefficient $\alpha$ and the kinematic viscosity $v$. The solid lines represent a linear regression of the data points. 


\section{Thermal diffusion properties of sucrose-water solutions.}

\section{Influence of the temperature and sugar concentration}

The disaccharide sucrose was selected in order to analyze the temperature and concentration dependence of the thermal diffusion properties. The same analysis had been performed with the monosaccharide, glucose. ${ }^{2}$ The values of the thermal diffusion properties are tabulated in Table 3 .

Figure 5 shows an increase of $S_{\mathrm{T}}, D_{\mathrm{T}}$ and $D$ with increasing temperature for all sugar concentrations. The same behavior has recently been found for aqueous solutions of the monosaccharide glucose ${ }^{2}$ and for the polysaccharide pullulan. ${ }^{29}$ A similar trend of the Soret coefficient with temperature has been found in protein and polypeptide solutions ${ }^{30-33}$ and in aqueous solution of the polysaccharide dextran. ${ }^{29}$ Piazza and coworkers suggested describing the temperature dependence of $S_{\mathrm{T}}$ by the following empirical equation ${ }^{30}$

$$
S_{\mathrm{T}}(T)=S_{\mathrm{T}}^{\infty}\left[1-\exp \left(\frac{T_{i n v}-T}{T_{0}}\right)\right]
$$

where $S_{\mathrm{T}}^{\infty}$ represents a high-T thermophobic limit, $T_{i n v}$ is the temperature where $S_{\mathrm{T}}$ changes sign, and $T_{0}$ represents the strength of temperature effects.

Table 5: Values of $S_{\mathrm{T}}^{\infty}, T_{i n v}$, and $T_{0}$ obtained from Eq. (3)

\begin{tabular}{|c|c|c|c|}
\hline c & $\begin{array}{c}S_{\mathrm{T}}^{\infty} \\
/ 10^{-3} \mathrm{~K}^{-1}\end{array}$ & $\begin{array}{l}T_{i n v} \\
/{ }^{\circ} \mathrm{C}\end{array}$ & $\begin{array}{c}T_{0} \\
/ \mathrm{K}\end{array}$ \\
\hline 0.1 & $5.27 \pm 0.02$ & $20.87 \pm 0.01$ & $32.0 \pm 0.1$ \\
\hline 0.2 & $5.37 \pm 0.05$ & $20.96 \pm 0.02$ & $33.0 \pm 0.4$ \\
\hline 0.4 & $4.94 \pm 0.01$ & $25.39 \pm 0.01$ & $33.1 \pm 0.1$ \\
\hline 0.6667 & $3.1 \pm 0.3$ & $50.3 \pm 0.1$ & $39.8 \pm 3$ \\
\hline
\end{tabular}

There is a change in sign of the Soret coefficient with temperature, which depends on the sugar concentration. Using equation Eq. (3) the sign change temperature shifts from $20.9^{\circ} \mathrm{C}$ to $50.3^{\circ} \mathrm{C}$ with increasing concentration. The negative Soret coefficients at low temperatures are probably related to bad solvent conditions. This has already been observed earlier for polyvinyl alcohol in water and polyethylenoxide in ethanol/water mixtures with a high ethanol content. ${ }^{34,35}$ In our case, 
the higher sugar content is close to the solubility limit so that a more negative $S_{\mathrm{T}}$ can be expected. In contrast to many other aqueous mixtures the concentration at which the sign change occurs is not independent of the temperature. ${ }^{36}$

Concerning the thermal diffusion coefficient, one can observe that $D_{\mathrm{T}}$ increases with increasing temperature. A similar trend has been also found in many systems, for instance, in aqueous suspensions of polystyrene spheres for different sizes of particles, ${ }^{25}$ in water and in water/ethanol solutions of polyethylene oxide, ${ }^{37}$ and in aqueous solutions of dextran. ${ }^{24}$ At low temperatures $\left(\mathrm{T}=15-20{ }^{\circ} \mathrm{C}\right) D_{\mathrm{T}}$ is practically independent of the mass concentration, while the differences become higher when increasing both the temperature and the sugar concentration. In other words, the higher the sugar concentration, the less pronounced is the increase of $D_{\mathrm{T}}$ with temperature (cf. Figure 5), and at the same time, the higher the temperature the more pronounced is the decrease of $D_{\mathrm{T}}$ with the sugar concentration (cf. Figure 6).

There is a linear increase in the mass diffusion coefficient with temperature, the gradient of which decreases with increasing sugar concentration. At $20{ }^{\circ} \mathrm{C}$ the error bars of the diffusion coefficient are considerably bigger compared to other temperatures. The reason is that measurements are close to sign changes. Therefore, the determination of the time constant, $\tau$, becomes more difficult because in the heterodyne signal the gap between the thermal plateau and concentration plateau is small. This is also the reason for the large uncertainties in the transport properties of maltose in water at $20^{\circ} \mathrm{C}$ (cf. Table 4).

The thermal diffusion, mass diffusion and Soret coefficients are plotted as function of the sucrose weight fraction in Figure 6. $D_{\mathrm{T}}$ shows a linear dependence on the sugar concentration, the slope of which varies as function of temperature. The slope is roughly zero at low temperatures, which means that in this case $D_{\mathrm{T}}$ is independent of the sugar content, and the slope increases for higher temperatures. The mass diffusion coefficient of sucrose decreases with increasing the sugar content, as in the case of glucose. ${ }^{2}$ The extrapolation of our data to diluted solutions agrees with the results of Mogi et al., ${ }^{38}$ who measured the mass diffusion coefficient of diluted sucrose solutions at different temperatures. For instance, at $15^{\circ} \mathrm{C}$, the quadratic extrapolation for dilute solution of our 


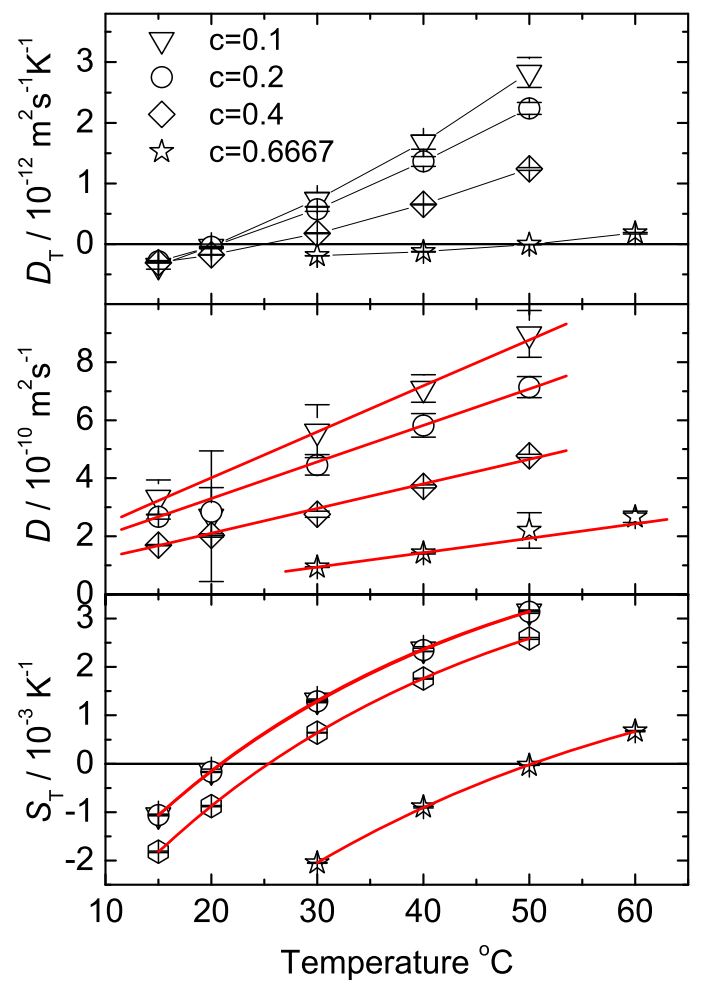

Figure 5: (color online) Temperature dependence of the thermal diffusion, mass diffusion and Soret coefficients of sucrose/water mixtures with sugar weight fractions $c=0.1,0.2,0.4$ and 0.6667 . The temperature dependence of $D$ and $S_{\mathrm{T}}$ has been fitted by linear regression and Eq. (3), respectively. 
diffusion coefficient provides $D=4.12 \times 10^{-10} \mathrm{~m}^{2} \mathrm{~s}^{-1}$ compared to the extrapolated value of Mogi et al. of $D=4.14 \times 10^{-10} \mathrm{~m}^{2} \mathrm{~s}^{-1}$. Furthermore, Gladden and Mole ${ }^{39}$ measured the mass diffusion coefficient of sucrose in water at a sugar weight fraction of $c=0.4096$ at $25^{\circ} \mathrm{C}$, with a result of $D=2.30 \times 10^{-10} \mathrm{~m}^{2} \mathrm{~s}^{-1}$. Our extrapolated data to $25^{\circ} \mathrm{C}$ and $c=0.4$ is $D=2.38 \times 10^{-10} \mathrm{~m}^{2} \mathrm{~s}^{-1}$.

For low concentrations $(c=0.1-0.2)$ the Soret coefficient is constant, while $S_{\mathrm{T}}$ decreases for higher concentrations (cf. Figure 6). Similar observations have been made for polystyrene in toluene. ${ }^{40} \mathrm{~A}$ possible explanation could be that the packing of the sugar molecules reaches a threshold concentration $\left(c^{*}\right)$, where the sugar-sugar interactions become more important. An estimation of $c^{*}$ could be done by determining the number of water molecules necessary to enclose a sugar molecule. For that, we determined the volume of the sucrose and water molecules by means of the Van der Waals increments. ${ }^{41}$ Assuming that both molecules are spherical, the radius of the sucrose and water are $R_{S}=3.8 \AA$ and $R_{w}=1.7 \AA$, respectively. The shell surrounding a sucrose molecule consists of 65 water molecules, which corresponds to a sucrose mass ratio of $c^{*}=0.22$. this concentration agrees with our experimental observation.

We also measured the viscosity and thermal expansion of the aqueous sucrose solutions at different concentrations and temperatures (see Table 1), in order to investigate whether the thermal diffusion coefficient depends linearly on the ratio $\alpha / v$. As can be seen in Figure 7, in general, the thermal diffusion coefficient increases with the ratio of $\alpha / \nu$. For a fixed concentration the behavior is linear as function of the temperature. If we increase the concentration at a fixed temperature, $D_{\mathrm{T}}$ decays first linearly. For the highest concentration $(c=0.6667)$ deviations from the linear behavior can be found. The temperature dependence is less pronounced for the high concentration and all data seem to converge to one point. A possible reason for the deviation from the linear behavior at high sugar content could be the increased dominance of sugar-sugar interaction. 


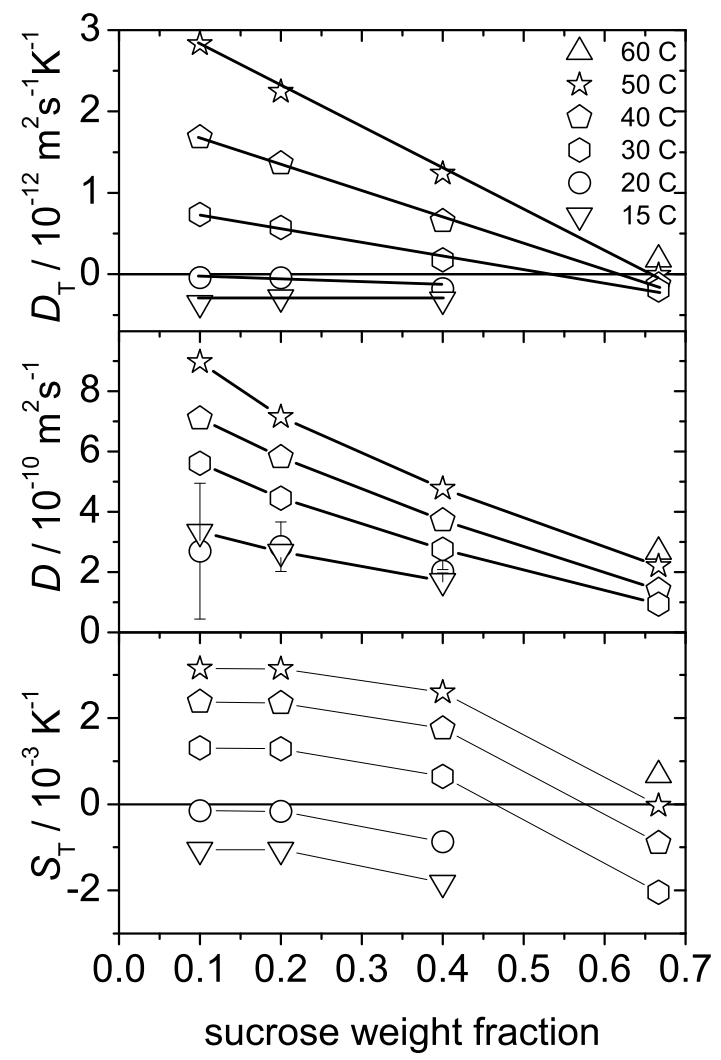

Figure 6: Concentration dependence of the thermal diffusion, mass diffusion and Soret coefficients of sucrose/water mixtures at different temperatures.

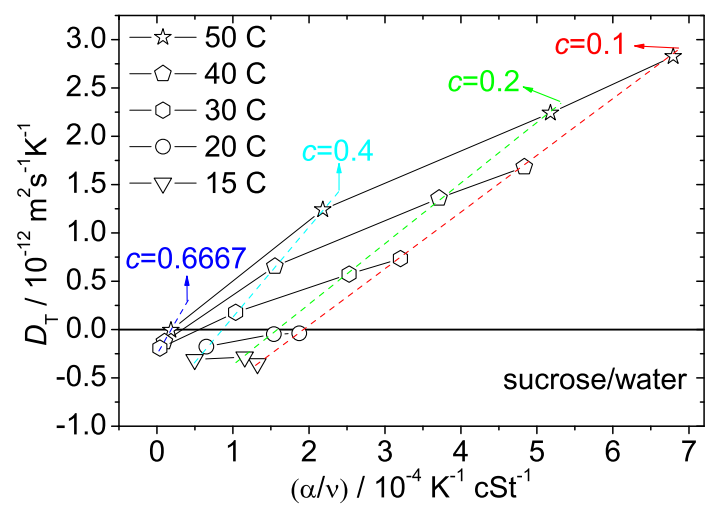

Figure 7: (color online) Thermal diffusion coefficient of sucrose aqueous solutions with a sugar weight fraction $c=0.1,0.2,0.4$ and 0.6667 at temperatures from 15 to $50{ }^{\circ} \mathrm{C}$ as a function of the ratio of the thermal expansion coefficient $\alpha$ and the kinematic viscosity $v$. 


\section{Thermal diffusion properties of different oligosaccharide-water solutions: in- fluence of the temperature}

Figure 8 shows a comparison of the thermal diffusion properties for the six aqueous oligosaccharide solutions at a weight fraction of $c=0.1$ as function of temperature. $D_{\mathrm{T}}$ increases with increasing temperature and decreasing molar mass of the sugar molecule. As mentioned before, $D_{\mathrm{T}}$ seems to be correlated with the solubility of the sugars, which also increases with temperature and decreases with the molar mass of the sugar molecules. For all studied temperatures, larger (heavier) sugar molecules have a stronger tendency to enrich at the cold side.

As expected, we find the slowest diffusion for the sugar molecule with the highest molar mass and $D$ increases with temperature (cf. Figure 8). On the other hand, the higher the temperature is, the higher is the difference in the mass diffusion coefficient between the different groups of oligosaccharides. The measurements at $20{ }^{\circ} \mathrm{C}$ have large uncertainties due to the proximity to the sign change temperature. This leads to a very small amplitude of the concentration plateau of the heterodyne signal, so that it is difficult to determine the diffusion constant.

The magnitude of the Soret coefficient becomes larger with increasing molecular weight. Although the stachyose has the lowest tendency to go to the warm, at high temperatures it has the highest $S_{\mathrm{T}}$ because of the small mass diffusion coefficient. Braibanti et al. ${ }^{25}$ made the same observation in different sizes of spherical polystyrene particle suspensions. At a temperature around 35 ${ }^{\circ} \mathrm{C}$, the Soret coefficient is virtually independent of the size of the molecule. The temperature at which the sugar changes the tendency of going from warm to cold, or vice versa, depends on the size of the sugar molecule. For disaccharides, it is on average at around $20{ }^{\circ} \mathrm{C}$. For trisaccharides, it is at about $24{ }^{\circ} \mathrm{C}$ and for the tetrasaccharide it is slightly higher, around $25{ }^{\circ} \mathrm{C}$.

\section{Conclusion}

In this study the thermal diffusion properties of aqueous solutions of oligosaccharides are presented at different temperatures and sugar concentrations. The study extends a recent study of 


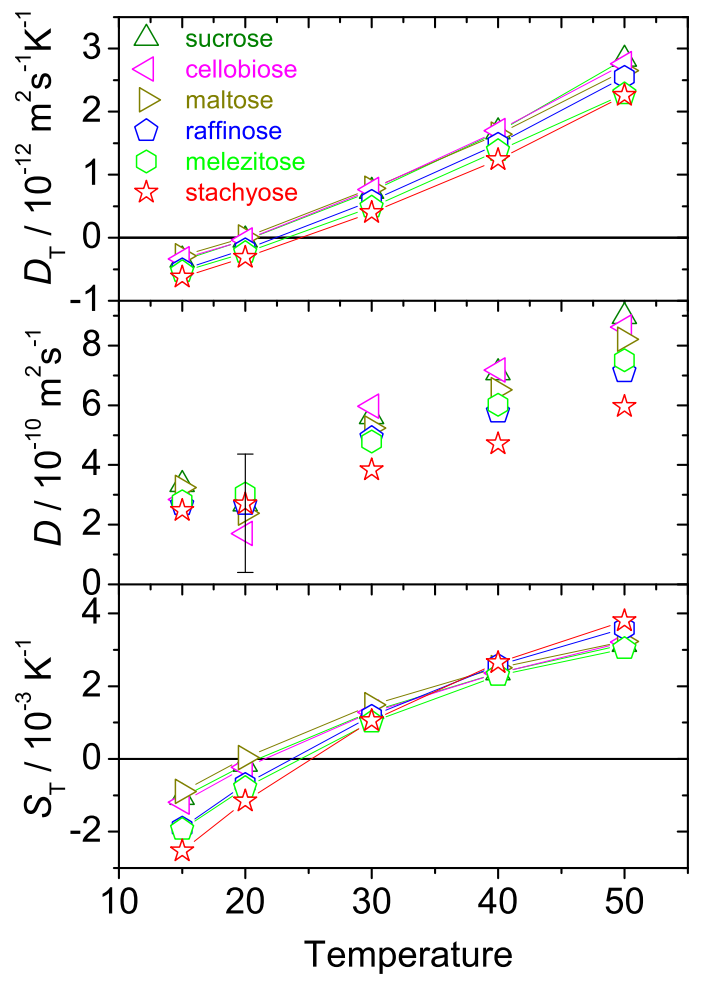

Figure 8: (color online) Thermal diffusion coefficient of different aqueous sugar solutions with a sugar weight fraction of $c=0.1$ at temperatures from 15 to $50{ }^{\circ} \mathrm{C}$. We indicated only the error bars for $D$ close to the sign change temperature. The other error bars are in the order of the symbol size. 
aqueous solutions of monosaccharides to longer and heavier sugar molecules. Within the range of temperatures considered, the thermal and mass diffusion coefficients are roughly constant for a fixed temperature and number of monomers, as in the case of monosaccharides. Therefore, the molecular structure of the monomers does not affect the mass diffusion.

Thermal and mass diffusion coefficients decrease with increasing size of the sugar molecule. While the Soret coefficient at low temperatures $\left(15-30^{\circ} \mathrm{C}\right)$ decreases with the number of monomers (cf. Figure 3), it remains practically constant at higher temperatures $\left(40-50{ }^{\circ} \mathrm{C}\right)$ (cf. Figure 2$)$.

In general, the thermal diffusion properties increase (decrease) with increasing temperature (concentration), independently of the sugar concentration (temperature). The Soret coefficient undergoes a sign change depending on the temperature and sugar concentration. A sign change of the Soret coefficient for low concentrations of sucrose solutions $(c<0.2)$ has been found at temperatures around $20{ }^{\circ} \mathrm{C}$, whereas this transition temperature is about $50{ }^{\circ} \mathrm{C}$ for more concentrated solutions $(c=0.6667)$.

The thermal diffusion coefficient increases with the ratio of thermal expansion coefficient and kinematic viscosity for the studied aqueous sugar solutions, as in the case of monosaccharide aqueous solutions. The same behavior occurs in the sucrose/water system when the temperature is fixed and the concentration of the sugar is changed. Additionally, this behavior is linear in the same system when the concentration is fixed and the temperature is changed.

\section{Acknowledgement}

We appreciate many fruitful discussions with Stefan Immel, Rio Kita and Michael Klein and thank Simon Rogers for careful reading the manuscript. Additionally, we thank the Department of Education, Universities and Investigation of Basque Government for the grant BFI08188.0. Financial support due to the Deutsche Forschungsgemeinschaft grant Wi 1684 is gratefully acknowledged. 


\section{References}

(1) Wiegand, S.; Köhler, W. Measurement of Transport Coefficients by an optical grating technique. In Thermal Nonequilibrium Phenomena in Fluid Mixtures; Springer: Berlin, 2002; Vol. LNP584, pp 189-210.

(2) Blanco, P.; Wiegand, S. J. Phys. Chem. B 2010, 114, 2807-2813.

(3) Polyakov, P.; Luettmer-Strathmann, J.; Wiegand, S. J. Phys. Chem. B 2006, 110, 2621526224.

(4) Blanco, P.; Bou-Ali, M. M.; Platten, J. K.; Urteaga, P.; Madariaga, J. A.; Santamaria, C. J. Chem. Phys. 2008, 129, 174504.

(5) Blanco, P.; Polyakov, P.; Bou-Ali, M. M.; Wiegand, S. J. Phys. Chem. B 2008, 112, 83408345.

(6) Blanco, P.; Bou-Ali, M. M.; Platten, J. K.; Madariaga, J. A.; Urteaga, P.; Santamaria, C. J. Non-Equilib. Thermodyn. 2007, 32, 309-317.

(7) Leahy-Dios, A.; Zhuo, L.; Firoozabadi, A. J. Phys. Chem. B 2008, 112, 6442-6447.

(8) Leahy-Dios, A.; Firoozabadi, A. J. Phys. Chem. B 2007, 111, 191-198.

(9) Ecenarro, O.; Madariaga, J. A.; Navarro, J. L.; Santamaria, C. M.; Carrion, J. A.; Saviron, J. M. Macromolecules 1994, 27, 4968-4971.

(10) Würger, A. Phys. Rev. Lett. 2009, 102, 078302.

(11) Stadelmaier, D.; Köhler, W. Macromolecules 2009, 42, 9147-9152.

(12) Stadelmaier, D.; Köhler, W. Macromolecules 2008, 41, 6205-6209.

(13) Osborn, H.; Khan, T. Oligosaccharides. Their synthesis and biological roles; Oxford University Press, New York, 2000. 
(14) Wiegand, S.; Ning, H.; Kriegs, H. J. Phys. Chem. B 2007, 111, 14169-14174.

(15) Thyagarajan, K.; Lallemand, P. Opt. Commun. 1978, 26, 54-57.

(16) Köhler, W. J. Chem. Phys. 1993, 98, 660-668.

(17) Köhler, W.; Rossmanith, P. J. Phys. Chem. 1995, 99, 5838-5847.

(18) Köhler, W.; Schäfer, R. Polymer analysis by thermal-diffusion forced Rayleigh scattering. In New Developments in Polymer Analytics II; Schmidt, M., Ed.; Springer: Berlin, 2000; Vol. 151, pp $1-59$.

(19) Leppla, C.; Wiegand, S. Philos. Mag 2003, 83, 1989-1999.

(20) Ning, H.; Kita, R.; Kriegs, H.; Luettmer-Strathmann, J.; Wiegand, S. J. Phys. Chem. B 2006, $110,10746-10756$.

(21) Wittko, G.; Köhler, W. Philos. Mag. 2003, 83, 1973-1987.

(22) Duhr, S.; Braun, D. PNAS 2006, 103, 19678-19682.

(23) Yan, Y.; Blanco, P.; Saghir, M. Z.; Bou-Ali, M. M. J. Chem. Phys. 2008, 129, 194507.

(24) Sugaya, R.; Wolf, B. A.; Kita, R. Biomacromolecules 2006, 7, 435-440.

(25) Braibanti, M.; Vigolo, D.; Piazza, R. Phys. Rev. Lett. 2008, 100, 108303.

(26) Brown, C. J. J. Chem. Soc. A 1966, 927-932.

(27) Semenov, S.; Schimpf, M. Phys. Rev. E 2004, 69, 011201.

(28) Brenner, H. Phys. Rev. E 2006, 74, 036306.

(29) Kishikawa, Y.; Wiegand, S.; Kita, R. Biomacromolecules 2010, 11, 740-747.

(30) Iacopini, S.; Rusconi, R.; Piazza, R. Eur. Phys. J. E 2006, 19, 59-67. 
(31) Piazza, R.; Iacopini, S.; Triulzia, B. Phys. Chem. Chem. Phys. 2004, 6, 1616-1622.

(32) Iacopini, S.; Piazza, R. Europhys. Lett. 2003, 63, 247-253.

(33) Piazza, R.; Parola, A. J. Phys.: Condens. Matter 2008, 20, 153102.

(34) Giglio, M.; Vendramini, A. Phys. Rev. Lett. 1977, 38, 26-30.

(35) de Gans, B.-J.; Kita, R.; Wiegand, S.; Luettmer Strathmann, J. Phys. Rev. Lett. 2003, 91, 245501.

(36) Polyakov, P.; Wiegand, S. J. Chem. Phys. 2008, 128, 0345005.

(37) Kita, R.; Wiegand, S.; Luettmer-Strathmann, J. J. Chem. Phys. 2004, 121, 3874-3885.

(38) Mogi, N.; Sugai, E.; Fuse, Y.; Funazukuri, T. J. Chem. Eng. Data 2007, 52, 40-43.

(39) Gladden, J. K.; Dole, M. J. Am. Chem. Soc. 1953, 75, 3900-3904.

(40) Rauch, J.; Köhler, W. J. Chem. Phys. 2003, 119, 11977-11988.

(41) Edward, J. T. J. Chem. Educ. 1970, 47, 261-270. 